\title{
Stress alters the expression of aquaporins in cultured rat intestinal epithelial cells
}

\author{
JIAN-AN CHEN ${ }^{1,2}$, LI-REN CHANG ${ }^{2}$, GUAN-MING FENG $^{2}$, SHU-TING LEE $^{3}$, \\ CHIEN-YAN HSIEH ${ }^{3}$, SENG-FENG JENG $^{2}$ and WEN-SHYAN HUANG ${ }^{4,5}$ \\ ${ }^{1}$ Division of Plastic Surgery, Kaohsiung Armed Forces General Hospital, Kaohsiung 802, Taiwan; \\ ${ }^{2}$ Department of Plastic Surgery, E-DA Hospital, Kaohsiung 824, Taiwan; ${ }^{3}$ Department of Biotechnology, \\ National Kaohsiung Normal University, Kaohsiung 802, Taiwan; ${ }^{4}$ Division of Plastic Surgery, \\ Kaohsiung Armed Forces General Hospital Zuoying Branch, Kaohsiung 813, Taiwan; \\ ${ }^{5}$ Department of Surgery, National Defense Medical Center, Taipei 114, Taiwan, R.O.C.
}

Received November 20, 2014; Accepted September 1, 2015

DOI: $10.3892 /$ etm.2015.2771

\begin{abstract}
Aquaporins (AQPs) are widely-expressed small water channel proteins that provide the major route for water transport across plasma membranes in various cell types. Although the quantity of water transported in the intestinal tract is second only to that in the kidney, the precise role of AQPs in this organ remains largely uncertain. The present study reports the effects of hypertonic stress and ischemia/reperfusion injury on the expression of AQPs in intestinal epithelial cells. Cultured rat intestinal epithelial cells were incubated in $300 \mathrm{mM}$ mannitol-containing, hypertonic culture medium or subjected to simulated ischemia/reperfusion treatment. The cell viability was evaluated by MTT assay, and the expression of AQPs was determined by semi-quantitative reverse transcription polymerase chain reaction and western blotting. Despite reduced viability, the cells exposed to hypertonic stress for $16 \mathrm{~h}$ demonstrated enhanced expression of AQP1 mRNA and protein. AQP9 and glycosylated AQP11 proteins were also markedly upregulated. Ischemia alone did not affect the cell viability, but subsequent reperfusion significantly reduced viability. The mRNA expression levels of all the tested AQPs were not altered by ischemia alone or by ischemia/reperfusion; however, AQP8 protein was markedly reduced by ischemic injury. In addition, treatment with ischemia alone eradicated the normally-expressed, non-glycosylated AQP11 protein whilst inducing pronounced expression of the glycosylated form. These observations may indicate that AQPs function in the intestinal epithelia in response to stress.
\end{abstract}

Correspondence to: Dr Wen-Shyan Huang, Division of Plastic Surgery, Kaohsiung Armed Forces General Hospital Zuoying Branch, 553 Junxiao Road, Zuoying, Kaohsiung 813, Taiwan, R.O.C. E-mail: hwsh9@hotmail.com; putai1018@gmail.com

Key words: aquaporin, hypertonic stress, intestinal epithelial cells, ischemia/reperfusion, mannitol

\section{Introduction}

The longstanding theory that water passes through the intestinal epithelia using simple diffusion was reassessed following the identification of aquaporins (AQPs) (1). The current, established hypothesis posits that water can cross the epithelia either by a paracellular pathway across the tight junctions or by transcellular pathways, involving 3 mechanisms as follows: Simple diffusion across the lipid bilayer; cotransport with ions and solutes; and diffusion across AQPs (1-3). AQPs are a family of homologous, water channel proteins that provide a major route for osmotically-driven water movement across the plasma membrane in various cell types. Currently, 13 distinct subtypes of AQPs (AQPs 0-12) have been identified, which are functionally subdivided into orthodox AQPs (AQPs 1, 2, 4, 5 and 8 , which are primarily water selective), aquaglyceroporins (AQPs 3, 7, 9 and 10, which are permeable to neutral solutes including glycerol and other small solutes, in addition to water) and unorthodox AQPs (AQPs 6, 11 and 12, for which localization and functions remain undetermined) (4-7).

Specific physiological roles for a number of the AQPs have been established through phenotype analysis of AQP-knockout mice. Compared with other organs, the role of AQPs in the kidney has been extensively studied, in part as the kidney is the most important organ involved in the homeostasis of water in the body. Previous studies have reported the expression of AQPs at various sites throughout the kidney, and these are suggested to be important in the urinary concentrating mechanism (8). The quantity of fluid transported in the intestinal tract is second only to that in the kidney, but the role of AQPs in intestinal physiology is comparatively unexplored.

At least 9 AQP subtypes (AQPs 1, 3, 4, 5, 7, 8, 9, 10 and 11) have been localized to the mammalian intestine (9-14), as follows: AQP1 in the endothelia and lacteals (9); AQP3 at the basolateral membrane of the epithelial cells lining the villus tip in the small intestine (10); AQP4 at the basolateral membrane of the crypt epithelium; AQP5 in the apical membrane of secretory cells in the duodenal glands; AQP7 and AQP8 at the apical membrane of the small intestine and colon (11); AQP9 in goblet cells in the small intestine (10); AQP10 in the duodenum 
and jejunum $(12,13)$; and AQP11 in the human duodenum and colon (14). Based on the multiple expression sites of AQPs in the intestinal tract, it is reasonable to presume that AQPs may effect an important functional role in water transport; however, in previous studies, phenotypic analyses of mice lacking AQPs did not support this hypothesis. For example, the water content in cecal stool from AQP4-null mice was similar to that from wild-type mice (15), and only minor phenotypic differences between wild-type and AQP8-knockout mice have been reported with regard to intestinal fluid absorption and secretion (16), leaving the physiological role of AQPs in the intestine unresolved. However, evidence of AQP tissue expression without demonstrable physiological function does not rule out possible functional roles of AQPs in the intestine under stressed or diseased conditions, as reported in other tissues (17-19). To test this hypothesis, the present study investigated the effects of hypertonic stress and ischemia/reperfusion injury on the expression level of AQP mRNA and protein in cultured intestinal epithelial cells, using semi-quantitative reverse transcription polymerase chain reaction (RT-PCR) and western blot analyses, respectively.

\section{Materials and methods}

Chemicals. All chemicals were purchased from Sigma Chemical Company (St. Louis, MO, USA).

Cell culture and treatment. Rat intestinal epithelial cells (IEC-6; Bioresource Collection and Research Center, Hsinchu, Taiwan) were cultivated in Dulbecco's modified Eagle's medium (DMEM; Sigma-Aldrich, St. Louis, MO, USA) with high glucose $(4,500 \mathrm{mg} / \mathrm{l})$, containing $5 \%$ fetal bovine serum (FBS; Hyclone; GE Healthcare Life Sciences, Logan, UT, USA) and penicillin-streptomycin (1:100; Sigma-Aldrich). To study the effects of hypertonicity on AQP expression in IEC-6 cells, the cells were seeded in $10-\mathrm{cm}$ dishes and grown to confluence. Culture medium was then replaced with hypertonic medium and the cells were incubated for $16 \mathrm{~h}$. Hypertonic medium was made by addition of $300 \mathrm{mOsmol}$ mannitol in isosmolar DMEM. For ischemic treatment, the cells were seeded in $10-\mathrm{cm}$ dishes and grown to confluence in 5\% FBS-supplemented DMEM. The cells were then washed with phosphate-buffered saline (PBS) prior to ischemic treatment. In this method, the cultures were treated with FBS- and glucose-free DMEM and placed into a modular incubator chamber (Billups-Rothenberg, Del Mar, CA, USA) that was sealed and flushed with $95 \% \mathrm{~N}_{2} / 5 \% \mathrm{CO}_{2}$ for $30 \mathrm{~min}$ at a rate of $21 / \mathrm{min}$. The hypoxic chamber was then sealed tightly and placed in a $37^{\circ} \mathrm{C}$, humidified, $5 \% \mathrm{CO}_{2} / 95 \%$ air incubator for $8 \mathrm{~h}$. Using this method, the $\mathrm{O}_{2}$ concentration of the culture media was measured as 0.8-1.0\% using a Dissolved Oxygen Meter, Model 5509 (Lutron, Taipei, Taiwan); this was significantly lower than that of the normoxic cultures (5-6\%). At the completion of ischemic exposure, half of the cultures were removed from the chamber and processed for analysis as described later. For the simulated 'ischemia/reperfusion' treatment, the other half of the cultures were removed from the chamber, the ischemic medium was replaced with normal culture medium (DMEM with FBS and glucose) and the culture was maintained in the $95 \% \mathrm{~N}_{2} / 5 \% \mathrm{CO}_{2}$ incubator for the 2-h reperfusion period.
This study was approved by the Animal Ethics Committee of Kaohsiung Armed Forces General Hospital Zuoying Branch (ZAFGH-101-09; Kaohsiung, Taiwan).

MTT reduction assay. Cell viability at the end of each experiment was analyzed by a MTT reduction assay. This assay is based upon the capacity of mitochondrial enzymes to transform MTT to MTT formazan. Briefly, MTT stock solution (5 $\mathrm{mg} / \mathrm{ml}$ in PBS) was added to each well of the multi-well plate containing cells at a final concentration of $0.5 \mathrm{mg} / \mathrm{ml}$. Following incubation at $37^{\circ} \mathrm{C}$ for $2 \mathrm{~h}$, the medium was aspirated and an equal volume of dimethyl sulfoxide was added to each well in order to dissolve the reduced MTT formazan crystals. The absorbance of the color product at $570 \mathrm{~nm}$ against the 630-nm reference was measured using a microplate reader (Sunrise; Tecan Systems, Inc., San Jose, CA, USA). All experiments were performed in triplicate.

$R T-P C R$. Total RNA was isolated using TRIzol reagent (Invitrogen, Carlsbad, CA, USA) according to the manufacturer's instructions. Total RNA $(5 \mu \mathrm{g})$ was reverse transcribed with random primers obtained from a High Capacity cDNA Reverse Transcription Kit (Applied Biosystems, Foster City, CA, USA). The cDNA was then amplified using polymerase chain reaction with primers specific against various AQPs (Table I). The PCR products were electrophoresed in $1 \%$ agarose gels and the bands were visualized by ethidium bromide staining. Densitometric analysis was performed using Quantity One software (Bio-Rad Laboratories, Inc., Hercules, CA, USA) and corrected for loading using the $\beta$-actin gene.

Western blot analysis. The cells were harvested in ice-cold lysis buffer [50 mM Tris ( $\mathrm{pH} 8.0), 150 \mathrm{mM} \mathrm{NaCl}, 0.1 \%$ SDS, $1 \%$ NP-40 and $1 \mathrm{mM}$ phenylmethylsulfonyl fluoride] containing protease inhibitor cocktail and then ultrasonicated. The homogenate was centrifuged at 13,000 x g for $20 \mathrm{~min}$ and the supernatant collected. Protein concentrations of supernatants were determined. Samples of supernatants containing $100 \mu \mathrm{g}$ protein were separated by $12 \%$ SDS-polyacrylamide gel electrophoresis and transferred to Hybond-P polyvinylidene difluoride membranes (EMD Millipore, Billerica, MA, USA) by electroelution. After being blocked for $3 \mathrm{~h}$ with Tris-buffered saline containing 5\% skimmed milk powder and $0.1 \%$ Tween-20, the membranes were incubated overnight with rabbit polyclonal antibodies against AQPs 1, 3, 4, 7, 8, 9 and $11(2 \mu \mathrm{g} / \mathrm{ml}$ for each AQP; Alpha Diagnostic, TX, USA) or $\beta$-actin (Sigma-Aldrich), diluted in the blocking solution. The membranes were washed and incubated with horseradish peroxidase-conjugated secondary antibodies for $1 \mathrm{~h}$. Finally, the signals were detected by enhanced chemiluminescence detection kit (GE Healthcare Life Sciences, Shanghai, China). The chemiluminescent signal was captured by a BioSpectrum 500 imaging system (UVP, Inc., Upland, CA, USA).

Data analysis. All data are expressed as the mean \pm standard error of the mean. Statistical evaluations were made by Student's t-test, using SigmaStat Software (Jandel Scientific, San Rafael, CA, USA). P $<0.05$ was considered to indicate a statistically significant difference. 
Table I. Primers for AQP expression.

\begin{tabular}{ll}
\hline mRNA & \multicolumn{1}{c}{ Primer sequence (5'-3') } \\
\hline AQP1 & \\
F & CTGTGGTGGCTGAGTTCCTG \\
R & ATTTCGGCCAAGTGAGTTCTC \\
AQP3 & \\
F & CTGTGGTGGCTGAGTTCCTG \\
R & ATTTCGGCCAAGTGAGTTCTC \\
AQP4 & \\
F & TTGGACCAATCATAGGCGC \\
R & GTCAATGTCGATCACATGC \\
AQP7 & \\
F & GCTTCGTGGATGAGGTATT \\
R & ACTTATGGGTAGGGTAGGTTT \\
AQP8 & \\
F & GGTGGACACTTCAACCCTGC \\
R & CCCAGCCAGTAGATCCAATG \\
AQP9 & \\
F & ACAATGAGCTGCGTGTGGCC \\
R & GATGGACTCATGGCCTTTGCTG \\
AQP11 & CACTGCATAGGACCCACGACAGG \\
F & \\
R & \\
F & \\
\hline actin & \\
\hline
\end{tabular}

$\mathrm{AQP}$, aquaporin; F, forward; R, reverse.

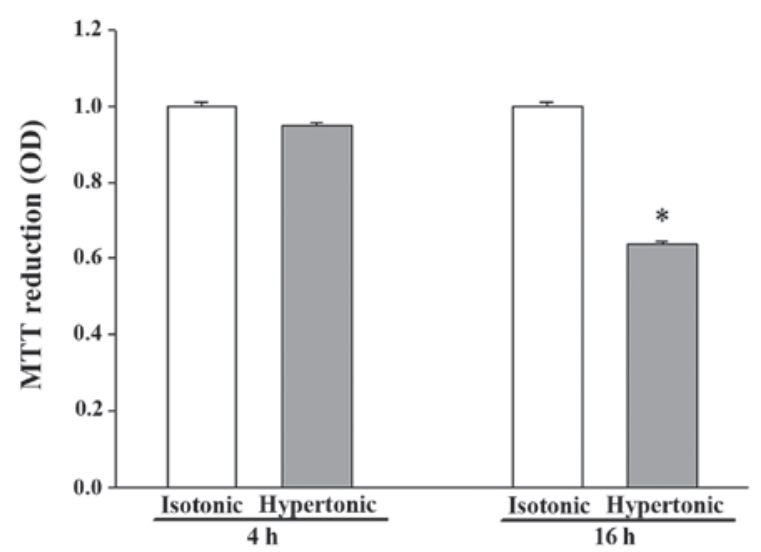

Figure 1. Effect of hypertonic stress on the viability of IEC-6 cells. Values presented are the mean \pm standard error of the mean from 3 experiments. ${ }^{*} \mathrm{P}<0.05$ vs. isotonic control. OD, optical density.

\section{Results}

Cell viability and $A Q P$ expression in response to hyperosmotic stress. Exposure to hypertonicity for $4 \mathrm{~h}$ did not significantly alter the viability of IEC- 6 cells, while $16 \mathrm{~h}$ of treatment resulted in apparent cell shrinkage and reduced viability (Fig. 1).
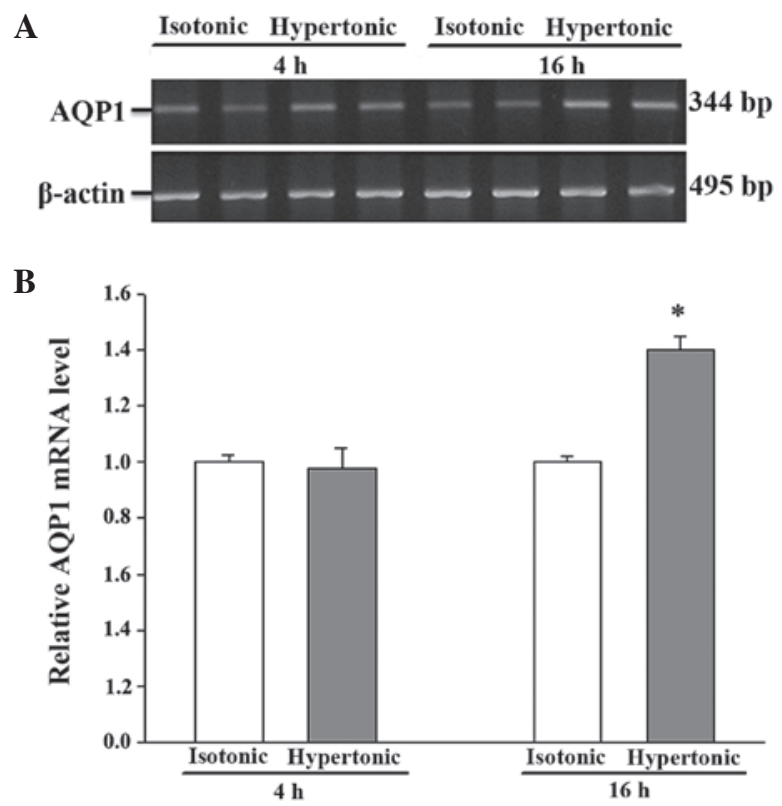

Figure 2. Reverse transcription polymerase chain reaction analysis of AQP1 mRNA expression in IEC-6 cells in response to hypertonic stress. The expression of AQP1 was normalized against that of the housekeeper gene, $\beta$-actin. (A) Representative gel; (B) densitometric analysis of AQP1 mRNA expression. The expression in the isotonic control condition is arbitrarily normalized to 1 . Results presented are the mean \pm standard error of the mean from 3 experiments. * $\mathrm{P}<0.05$ vs. control. AQP, aquaporin.

$\mathbf{A}$

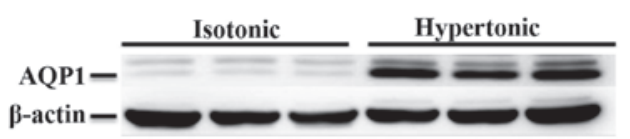

B

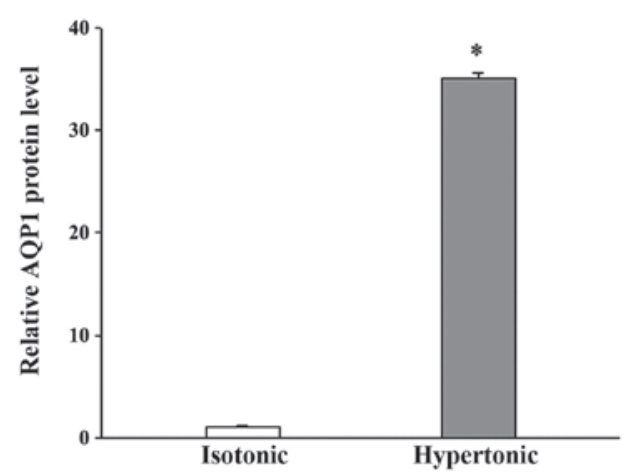

Figure 3. Western blot analysis of AQP1 protein expression in IEC-6 cells in response to $16 \mathrm{~h}$ of hypertonic exposure. The expression of AQP1 was normalized against that of $\beta$-actin. (A) Representative immunoblot; (B) densitometric analysis of AQP1 protein expression. Results presented are the mean \pm standard error of the mean from 3 experiments. ${ }^{*} \mathrm{P}<0.05$ vs. isotonic control. AQP, aquaporin.

There was no detectable difference in AQP1 mRNA abundance after $4 \mathrm{~h}$ in hypertonic media when compared to its abundance in isotonic (control) conditions (Fig. 2). Despite the moderate expression of AQP1 mRNA in isotonic conditions (Fig. 2), an extremely low level of AQP1 protein was noted (Fig. 3). After $16 \mathrm{~h}$ of hypertonic exposure, AQP1 mRNA expression was significantly enhanced (Fig. 2) and 
A

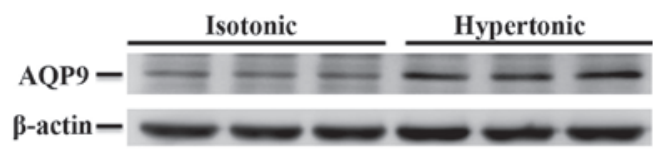

B

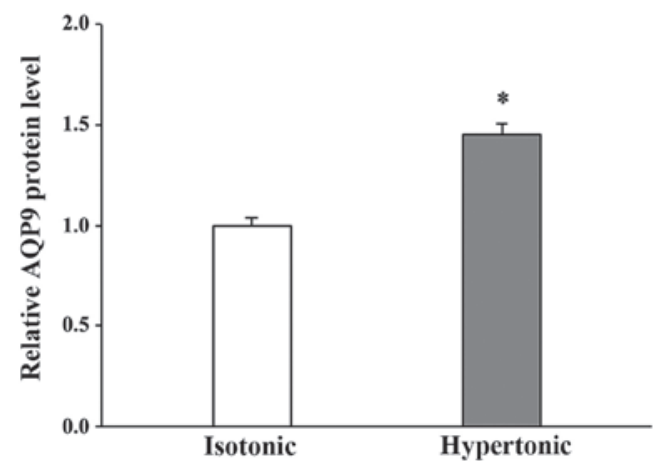

Figure 4. Western blot analysis of AQP9 protein expression in IEC-6 cells in response to $16 \mathrm{~h}$ of hypertonic exposure. The expression of AQP9 was normalized against that of $\beta$-actin. (A) Representative immunoblot; (B) densitometric analysis of AQP9 protein expression. Results presented are the mean \pm standard error of the mean from 3 experiments. $\mathrm{P}<0.05$ vs. isotonic control. AQP, aquaporin.

A

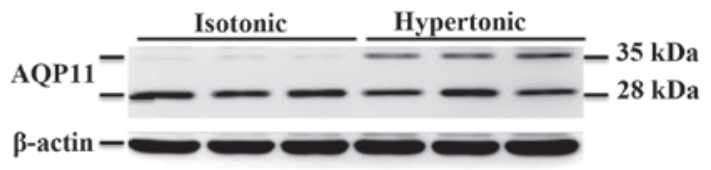

B

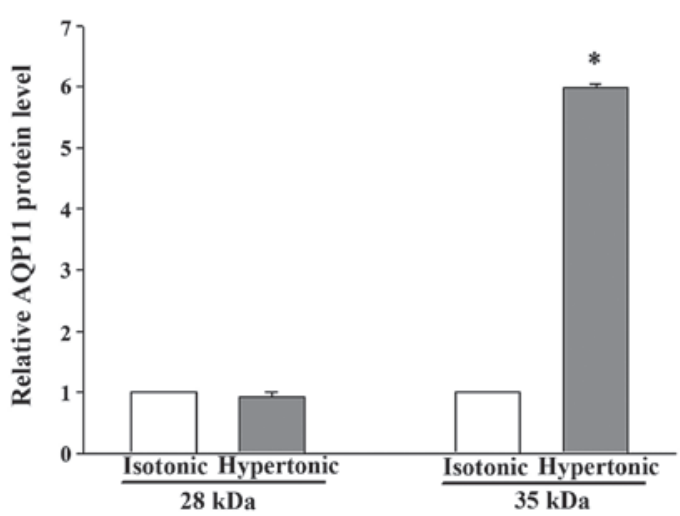

Figure 5. Western blot analysis of AQP11 protein expression in IEC-6 cells in response to $16 \mathrm{~h}$ of hypertonic exposure. The expression of AQP11 was normalized against that of $\beta$-actin. (A) Representative immunoblot; (B) densitometric analysis of AQP11 protein expression. Results presented are the mean \pm standard error of the mean from 3 experiments. ${ }^{~} \mathrm{P}<0.05$ vs. isotonic control. AQP, aquaporin.

protein expression was concomitantly elevated, undergoing a 37-fold increase over the expression levels observed in isotonic conditions (Fig. 3). The mRNA and protein expression level of AQPs 3, 4, 7 and 8 in response to 4 or $16 \mathrm{~h}$ of osmotic stress were similar to their corresponding control levels (data not shown). Osmotic stress did not affect the mRNA abundance of AQPs 9 and 11 (data not shown), but a significant increase in the expression level of the 2 proteins was observed in the cells exposed to hypertonic medium for $16 \mathrm{~h}$ (Figs. 4 and 5),

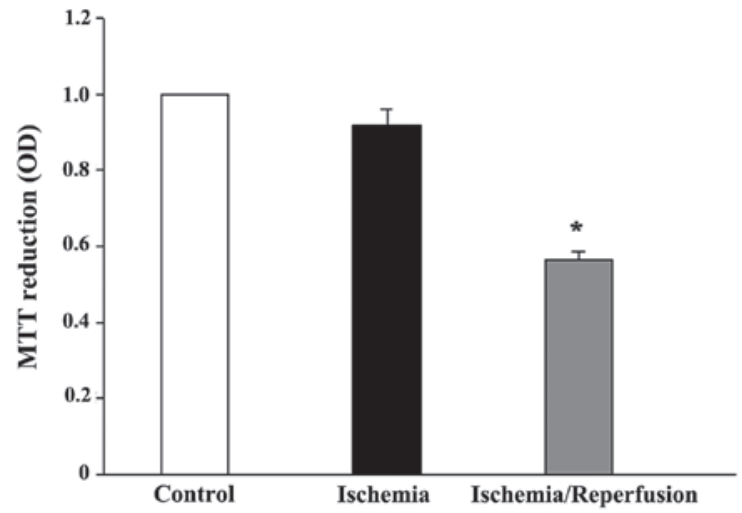

Figure 6. Effect of ischemia and ischemia/reperfusion on the viability of IEC- 6 cells. Values presented are the mean \pm standard error of the mean of at least 3 experiments. ${ }^{*} \mathrm{P}<0.05$ vs. isotonic control. OD, optical density.
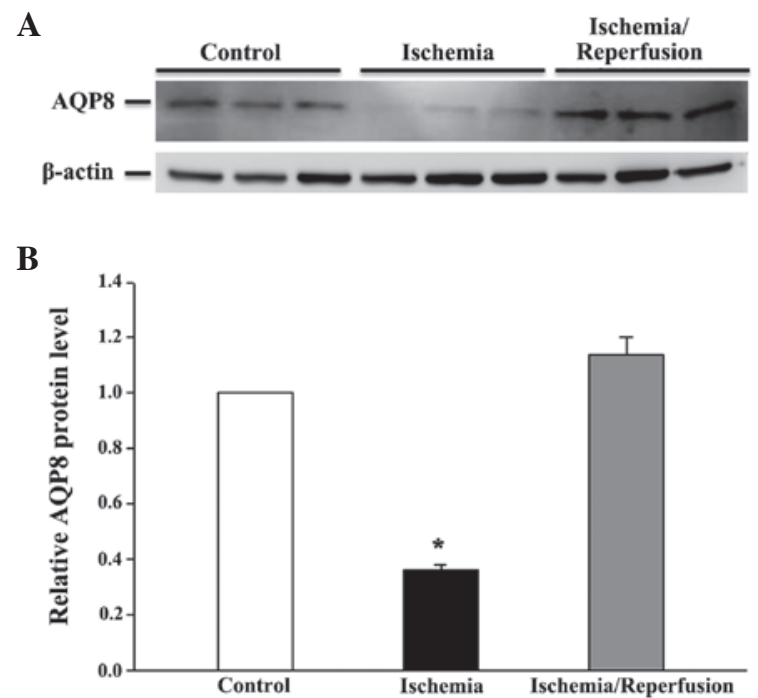

Figure 7. Western blot analysis of AQP8 protein expression in IEC-6 cells in response to ischemia, ischemia-reperfusion. The expression of AQP8 was normalized against that of $\beta$-actin. (A) Representative immunoblot; (B) densitometric analysis of AQP11 protein expression. Values presented are the mean \pm standard error of the mean from 3 experiments. ${ }^{*} \mathrm{P}<0.05$ vs. isotonic control. AQP, aquaporin.

which was not observed following a 4-h exposure (data not shown). Notably, the anti-AQP11 polyclonal antibody used in this study recognized 2 protein bands on immunoblots: One corresponded to the non-glycosylated form $(\sim 28 \mathrm{kDa})$, whereas the other was presumed to represent the glycosylated form ( $35 \mathrm{kDa})$ (20). Under isotonic conditions, the non-glycosylated AQP11 was markedly expressed; however, the glycosylated form was barely detectable (Fig. 5). After $16 \mathrm{~h}$ of osmotic stress, the expression of the 28-kDa AQP11 was unchanged, yet the glycosylated protein was markedly expressed. No significant alterations in the mRNA expression levels of AQP9 and AQP11 were observed (data not shown).

Cell viability and $A Q P$ expression in response to ischemia-reperfusion. While $8 \mathrm{~h}$ of ischemia exposure did not affect the cell viability (Fig. 6), it did induce a cell morphological change from a cuboidal shape to a smaller, thinner 
A

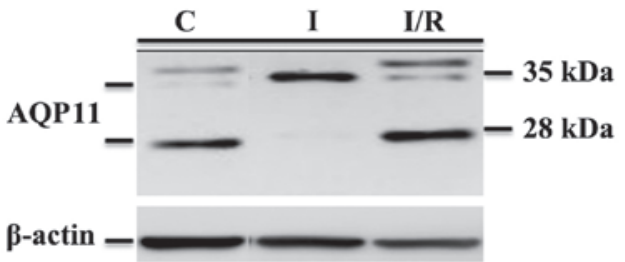

B

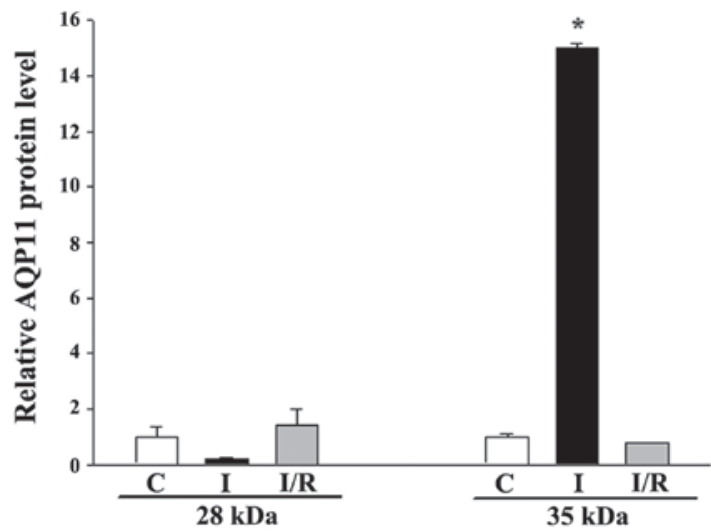

Figure 8. Western blot analysis of AQP11 protein expression in IEC-6 cells in response to ischemia and ischemia-reperfusion. The expression of AQP11 was normalized against that of $\beta$-actin. (A) Representative immunoblot; (B) densitometric analysis of AQP11 protein expression. Values presented are the mean \pm standard error of the mean of 3 experiments ${ }^{*} \mathrm{P}<0.05$ vs. isotonic control. AQP, aquaporin; C, control; I, ischemia $\mathrm{I} / \mathrm{R}$, ischemia-reperfusion

and loose cell-cell boundary appearance (data not shown). Subsequent reperfusion markedly reduced the cell viability, but restored the cell morphology. The mRNA expression levels of all the tested AQPs (AQP1, 3, 4, 7, 8, 9 and 11) were unaffected by ischemia alone or ischemia/reperfusion (data not shown). However, the protein expression of AQP8 and AQP11 was significantly altered ( $\mathrm{P}<0.05$ vs. control). AQP8 was downregulated by ischemia, but returned to the control level following reperfusion (Fig. 7). Similarly, moderate expression of non-glycosylated AQP11 (28 kDa) and faint expression of the glycosylated protein $(35 \mathrm{kDa})$ were observed under control conditions (Fig. 8). Ischemia alone eradicated the expression of the 28-kDa AQP11 protein, but induced the pronounced expression of the $35-\mathrm{kDa}$ form. Following reperfusion, this ischemia-altered expression was restored to the normal profile of AQP11 expression.

\section{Discussion}

To the best of our knowledge, the present study demonstrated for the first time the differential AQP expression profiles in intestinal epithelial cells that were exposed to environmental stress.

Intestinal epithelial cells, and similarly renal epithelial cells, are exposed to extreme osmolar environments. Osmotic stress may exert detrimental effects on cells, triggering adaptation mechanisms to re-establish homeostasis through changes to cell structure and function $(21,22)$. In response to osmotic stress, a limited number of genes, including the AQP genes, are upregulated, despite an overall decrease in DNA synthesis,
RNA transcription and protein synthesis (23-25). Previous studies have documented the enhanced expression of AQPs 1, $2,3,4,5$ and 9 in various, osmotically-disturbed cell types (26). In the present study, the protein expression of AQP1, 9 and 11 was increased after $16 \mathrm{~h}$, but not $4 \mathrm{~h}$, of hypertonic exposure, suggesting that the elevated protein expression is a delayed response. Altered protein expression may be correlated with the observed cell shrinkage and reduced cell viability after $16 \mathrm{~h}$ of osmotic challenge, although the mechanisms involved are currently unknown.

In the intestinal tract, AQP1 is expressed in endothelia and lacteals (9), but has not previously been reported to exist in epithelial cells. In the current study, hypertonic stress increased AQP1 mRNA and AQP1 protein abundance, suggesting that activation occurs at the transcriptional and translational levels. Furthermore, upregulated AQP1 protein may be partially caused by the hypertonicity-induced increase in protein stability; a previous study has demonstrated that hypertonic stress decreases AQP1 ubiquitination and increases protein stability, thereby contributing to overall protein induction (27). An increase in protein stability may also contribute to the increased AQP9 and 11 protein expression, considering their unchanged mRNA level in response to hyperosmotic stress. This increased protein expression may also be due to a hypertonicity-induced increase in mRNA stability.

AQP9, in addition to its function as a water channel, is also permeable to lactate and a wide variety of neutral solutes, including $\beta$-hydroxybutyrate, glycerol, carbamides, purines, pyrimidines, urea, mannitol and sorbitol (28). In the present study, the upregulation of AQP9 protein in cells maintained in mannitol-containing medium for $16 \mathrm{~h}$ may suggest an involvement of AQP9 in the transmembrane transport of mannitol. However, the transport of mannitol was not measured in this experiment.

Glycosylated proteins are an established focus of study, and have been suggested to be involved in protein folding and stability, the transport of proteins through secretory pathways, molecular recognition processes and cell-cell interaction (29). A previous study reported that glycosylation increases the thermostability of human AQP10 (30); the functional significance for the expression of glycosylated AQP11 (35 kDa) after $16 \mathrm{~h}$ of osmotic challenge thus requires greater investigation.

The intestine is the most sensitive to ischemia/reperfusion injury amongst the internal organs (31). Despite the reduced viability in ischemia/reperfusion-treated cells, the mRNA expression of all the tested AQPs was unaffected by ischemia alone or ischemia/reperfusion, and only the expression of AQP8 and AQP11 proteins was altered in the present study. AQP8 was substantially downregulated in an ischemic state and returned to the control level following reperfusion, indicating that this is a reversible response. Similarly, ischemia alone induced the pronounced expression of glycosylated AQP11 protein, revealed alongside a comparative absence of the non-glycosylated form; this expression pattern was completely restored following reperfusion, indicating that this is also a reversible response.

In conclusion, although the functional significance for the altered expression of AQPs is currently unresolved, the observations presented in the current study may indicate a role of AQPs in the adjustment of the intestinal epithelia in response to stress. 


\section{Acknowledgements}

The present study was supported by a grant from Kaohsiung Armed Forces General Hospital Zuoying Branch, Taiwan (no. ZAFGH 101-09).

\section{References}

1. Loo DD, Zeuthen T, Chandy G and Wright EM: Cotransport of water by the $\mathrm{Na}^{+} /$glucose cotransporter. Proc Natl Acad Sci USA 93: 13367-13370, 1996.

2. Ma T and Verkman AS: Aquaporin water channels in gastrointestinal physiology. J Physiol 517: 317-326, 1999.

3. Masyuk AI, Marinelli RA and LaRusso NF: Water transport by epithelia of the digestive tract. Gastroenterology 122: 545-562, 2002.

4. Agre P, King LS, Yasui M, Guggino WB, Ottersen OP, Fujiyoshi Y, Engel $A$ and Nielsen S: Aquaporin water channels - from atomic structure to clinical medicine. J Physiol 542: 3-16, 2002.

5. Nielsen J, Kwon TH, Christensen BM, Frøkiaer J and Nielsen S: Dysregulation of renal aquaporins and epithelial sodium channel in lithium-induced nephrogenic diabetes insipidus. Semin Nephrol 28: 227-244, 2008.

6. Nielsen S, Frøkiaer J, Marples D, Kwon TH, Agre P and Knepper MA: Aquaporins in the kidney: From molecules to medicine. Physiol Rev 82: 205-244, 2002.

7. Rojek A, Praetorius J, Frøkiaer J, Nielsen S and Fenton RA: A current view of the mammalian aquaglyceroporins. Annu Rev Physiol 70: 301-327, 2008.

8. Kwon TH, Nielsen J, Møller HB, Fenton RA, Nielsen S and Frøkiær J: Aquaporins in the kidney. In: Aquaporins. Handbook of Experimental Pharmacology. Beitz E (ed). Vol 190 Springer-Verlag, Berlin, Heidelberg, pp95-132, 2009.

9. Marinelli RA, Tietz PS, Pham LD, Rueckert L, Agre P and LaRusso NF: Secretin induces the apical insertion of aquaporin-1 water channels in rat cholangiocytes. Am J Physiol 276: G280-G286, 1999.

10. Matsuzaki T, Tajika Y, Ablimit A, Aoki T, Hagiwara H and Takata K: Aquaporins in the digestive system. Med Electron Microsc 37: 71-80, 2004.

11. Laforenza U, Gastaldi G, Grazioli M, Cova E, Tritto S, Faelli A, Calamita $\mathrm{G}$ and Ventura U: Expression and immunolocalization of aquaporin-7 in rat gastrointestinal tract. Biol Cell 97: 605-613, 2005.

12. Hatakeyama S, Yoshida Y, Tani T, Koyama Y, Nihei K, Ohshiro K, Kamiie JI, Yaoita E, Suda T, Hatakeyama K and Yamamoto T: Cloning of a new aquaporin (AQP10) abundantly expressed in duodenum and jejunum. Biochem Biophys Res Commun 287: 814-819, 2001.

13. Ishibashi K, Morinaga T, Kuwahara M, Sasaki S and Imai M: Cloning and identification of a new member of water channel (AQP10) as an aquaglyceroporin. Biochim Biophys Acta 1576 335-340, 2002
14. Laforenza U: Water channel proteins in the gastrointestinal tract. Mol Aspects Med 33: 642-650, 2012.

15. Wang KS, Ma T, Filiz F, Verkman AS and Bastidas JA: Colon water transport in transgenic mice lacking aquaporin-4 water channels. Am J Physiol Gastrointest Liver Physiol 279: G463-G470, 2000.

16. Yang B, Song Y, Zhao D and Verkman AS: Phenotype analysis of aquaporin-8 null mice. Am J Physiol Cell Physiol 288: C1161-C1170, 2005.

17. Kwon TH, Hager H, Nejsum LN, Andersen ML, Frøkiaer J and Nielsen S: Physiology and pathophysiology of renal aquaporins. Semin Nephrol 21: 231-238, 2001.

18. Hara-Chikuma M and Verkman AS: Roles of aquaporin-3 in the epidermis. J Invest Dermatol 128: 2145-2151, 2008.

19. Umenishi F and Schrier RW: Identification and characterization of a novel hypertonicity-responsive element in the human aquaporin-1 gene. Biochem Biophys Res Commun 292: 771-775, 2002.

20. García F, Kierbel A, Larocca MC, Gradilone SA, Splinter P, LaRusso NF and Marinelli RA: The water channel aquaporin-8 is mainly intracellular in rat hepatocytes, and its plasma membrane insertion is stimulated by cyclic AMP. J Biol Chem 276: 12147-12152, 2001.

21. Kültz D and Burg M: Evolution of osmotic stress signaling via MAP kinase cascades. J Exp Biol 201: 3015-3021, 1998.

22. Schwartz LM and Osborne BA: Programmed cell death, apoptosis and killer genes. Immunol Today 14: 582-590, 1993.

23. Cohen DM, Wasserman JC and Gullans SR: Immediate early gene and HSP70 expression in hyperosmotic stress in MDCK cells. Am J Physiol 261: C594-C601, 1991.

24. Burg MB, Kwon ED and Kültz D: Osmotic regulation of gene expression. FASEB J 10: 1598-1606, 1996.

25. Blomberg, A: Osmoresponsive proteins and functional assessment strategies in Saccharomyces cerevisiae. Electrophoresis 18: 1429-1440, 1997.

26. Burg MB, Ferraris JD and Dmitrieva NI: Cellular response to hyperosmotic stresses. Physiol Rev 87: 1441-1474, 2007.

27. Leitch V, Agre P and King LS: Altered ubiquitination and stability of aquaporin-1 in hypertonic stress. Proc Natl Acad Sci USA 98: 2894-2898, 2001.

28. Tsukaguchi H, Shayakul C, Berger UV, Mackenzie B, Devidas S, Guggino WB, van Hoek AN and Hediger MA: Molecular characterization of a broad selectivity neutral solute channel. J Biol Chem 273: 24737-24743, 1998.

29. Sato Y and Endo T: Alteration of brain glycoproteins during aging. Geriatr Gerontol Int 10 (Suppl 1): S32-S40, 2010.

30. Öberg F, Sjöhamn J, Fischer G, Moberg A, Pedersen A, Neutze R and Hedfalk K: Glycosylation increases the thermostability of human aquaporin 10 protein. J Biol Chem 286: 31915-31923, 2011.

31. Sasaki M and Joh T: Oxidative stress and ischemia-reperfusion injury in gastrointestinal tract and antioxidant, protective agents. J Clin Biochem Nutr 40: 1-12, 2007. 